\title{
Experimental Teaching of Microelectronics Assembly Technology
}

\section{Ivan Szendiuch}

Brno University of Technology, Faculty of Electric Engineering and Communication, Department of Microelectronics, CZ-Technicka 10, 61600 Brno, Czech Republic

How to cite this paper: Szendiuch, I. (2020) Experimental Teaching of Microelectronics Assembly Technology. The Educational Review, USA, 4(2), 23-29.

http://dx.doi.org/10.26855/er.2020.02.001

*Corresponding author: Ivan Szendiuch, Brno University of Technology, Faculty of Electric Engineering and Communication, Department of Microelectronics, CZTechnicka 10, 61600 Brno, Czech Republic.

Email: szend@feec.vutbr.cz

\begin{abstract}
This contribution gives some practical experience about the education program in the microelectronics assembly technology, as part of the base of hardware. It is closed to modern technologies of electronic packaging and interconnection. The subject "Modern Microelectronics Technology" should be a par of the modern educational programs in the area of "Electronics Hardware" in both Bachelor's and Master's degree program. The content of the lectures is in accordance with the global development and is co-ordinated with programs at various universities worldwide. A new idea, and at the same time the main objective in this area is to achieve still closer contact of the University with the industry in terms of technological integration. Lectures and laboratory curricula are described, as well as the supporting project, which is implemented in the form of an integrated circuit, by students in team work organization. Practical training in the microelectronics laboratory forms a very important part of the learning process, which is positive valued by students, and was also awarded by the International Microelectronics Assembly and Packaging Society [1]. The laboratory is based on low-cost no-vacuum thick film technology, which allows students to realize various electronics components and also their own integrated circuit. Therefore, the main emphasis is on the acquisition of practical skills and abilities. It also increases students' motivation and their active approach to study. This paper describes the structure of the course and includes also some experience of teaching.
\end{abstract}

\section{Keywords}

Engineering education, Microelectronics, Electronics hardware, Interdisciplinary subject

\section{Introduction}

Microelectronics technology is a very important part of the practical electronics because it creates electronic hardware, which is now included in all industry areas. To have basic ideas about principle of hardware requires some theoretical and practical knowledge for all engineers. The importance of knowledge "hardware" is based on the fact that a large proportion of engineers are in their practice often in contact with electronics. Electronics are everywhere nowadays in various application areas, such as communication and control equipment, consumer sector, medical, automotive, computer science and many others. Well treated with hardware means to save costs, improve quality and also protect the environment and human health.

Microelectronics assembly and packaging technology is one of the emerging areas in today's electronics. There is an emerging global focus on this domain, inside both academia and industry. It is necessary to explore new learning opportunities that will support not only theoretical but also practical instruction. This requires the introduction of 
teaching new technical knowledge, both theoretical and experimental. Described is the innovated subject "Modern microelectronic technology", which is part of a programme of study at Brno University of Technology, Faculty of Electrical Engineering and Communication. The Masters study programme is two year programme concentrated on electronics engineering education. The following six branches comprise the programme:

- Microelectronics,

- Electronics, Communication and Informatics,

- Electrical Manufacturing and Management,

- Cybernetics, Control and Measurements,

- Biomedical and Ecological Engineering,

- Power Electrical Engineering.

Modern Microelectronics Technology, in other words "Electronics hardware" is optional subject for branches Microelectronics, Electronics and Communication, Automation and Measurement, Teleinformatics and Power Electrics. Represents advanced technology in terms of components and processes, including partial design rules. Its innovative content is provided below, including a new organization of practical training and links with practice.

\section{Fundamental terms and structure of electronics hardware}

The Merriam-Webster dictionary offers a definition of the term "technology" as "the practical application of knowledge especially in a particular area" and also "a capability given by the practical application of knowledge". Actually the frequently used term "high technology" can be understood as "scientific technology involving the production or use of advanced or sophisticated devices especially in the fields of electronics and computers". If we add the term "microelectronics", then particular area is exactly defined. Then we can express the following definition: "Microelectronics Technology for education process" is science giving knowledge about design, production, use and liquidation of electronics hardware".

The basis of progress in microelectronics assembly technology and also in electronics hardware is comprised immediately in microelectronics technology development, which is changing the approach of engineers in managing positions to decision-making during recent years. The progress in microelectronics assembly technology is strongly influenced by "technology integration" process that assumes different activity sectors together, as is illustrated in Fig. 1.

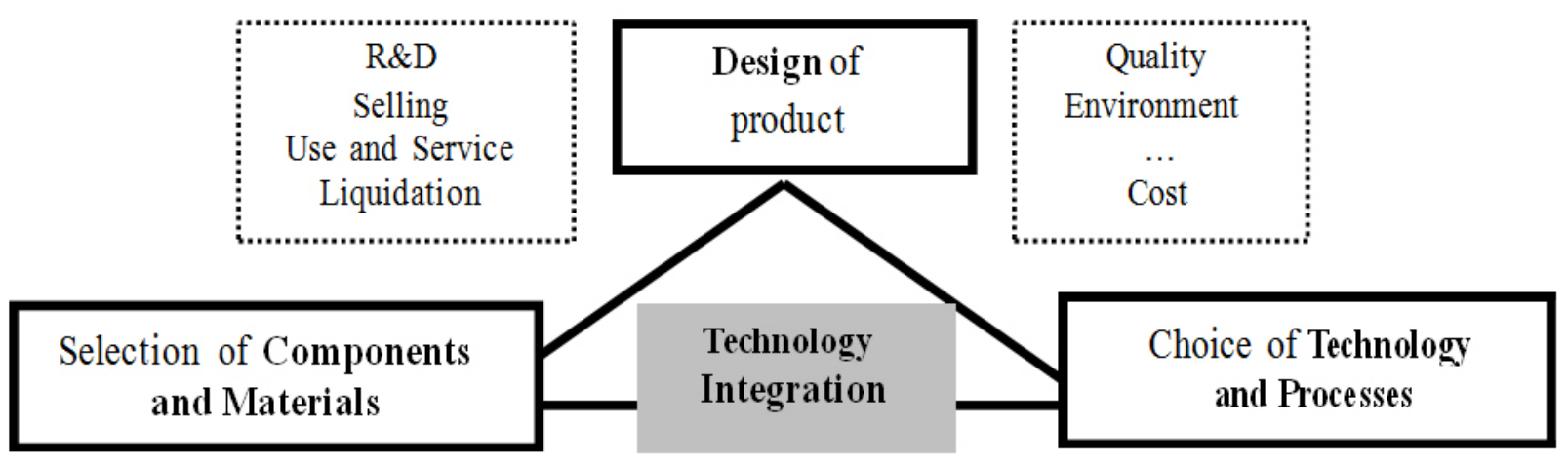

Figure 1. Explanation of the term "Technology integration"

Technological integration requires the basic knowledge of many formerly independent areas, as system engineering, material science, computer practicality, thermal management, quality and also environmental management etc Also selected rules of general management are necessary for good understanding and decision-making ability in this area. The development of new products is more complex and sophisticated and asks approach that is ever more open than in the past time. Also knowledge of electronics hardware becomes highly interdisciplinary area containing different sectors as could be seen in Fig. 2. 


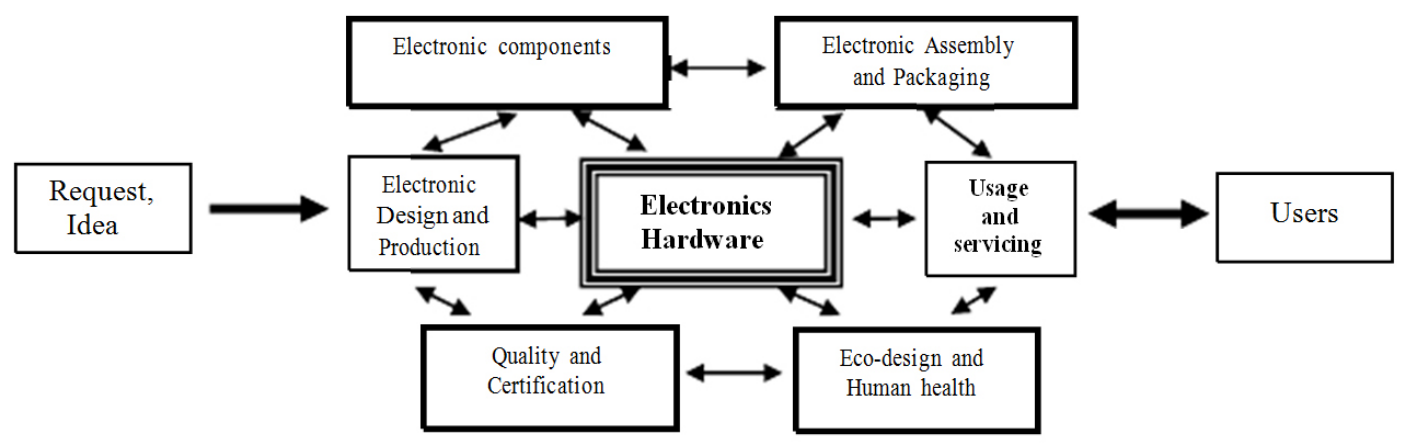

Figure 2. Main segments of electronics hardware in the context of technological integration

Electronics hardware consists of multiple areas of expertise that are logically connected. In the microelectronics assembly technology sector increasingly important core area of knowledge, which deals with electronics hardware is "Electronics Packaging and Interconnection" [2]. This area becomes to be more and more complex and includes more subjects.

The fundamental part of today's Microsystems is the semiconductor chip. To realize some functions or activity other apparatuses are also necessary, such as heart, hands, legs and all the body with sensing and executive parts. In electronics system the package is like the body, sensors as receptors, blood as electrical current, heart as source etc. Development of semiconductor chips has made enormous strong progress in the end of last centur and this continues without end. The same situation didn't set by packaging, interconnection and assembly technology processes in. That means there is strong need to push assembly technology more intensive to achieve necessary technical level.

Pyramid schema giving more concrete overview of knowledge in the area of electronics hardware is shown in Fig. 3. The "core" thematic topics for electronics packaging are labeled using strong frame. An optimum and effective range of individual topics is achieved by including practical laboratory training, and external cooperation with research and production entities.

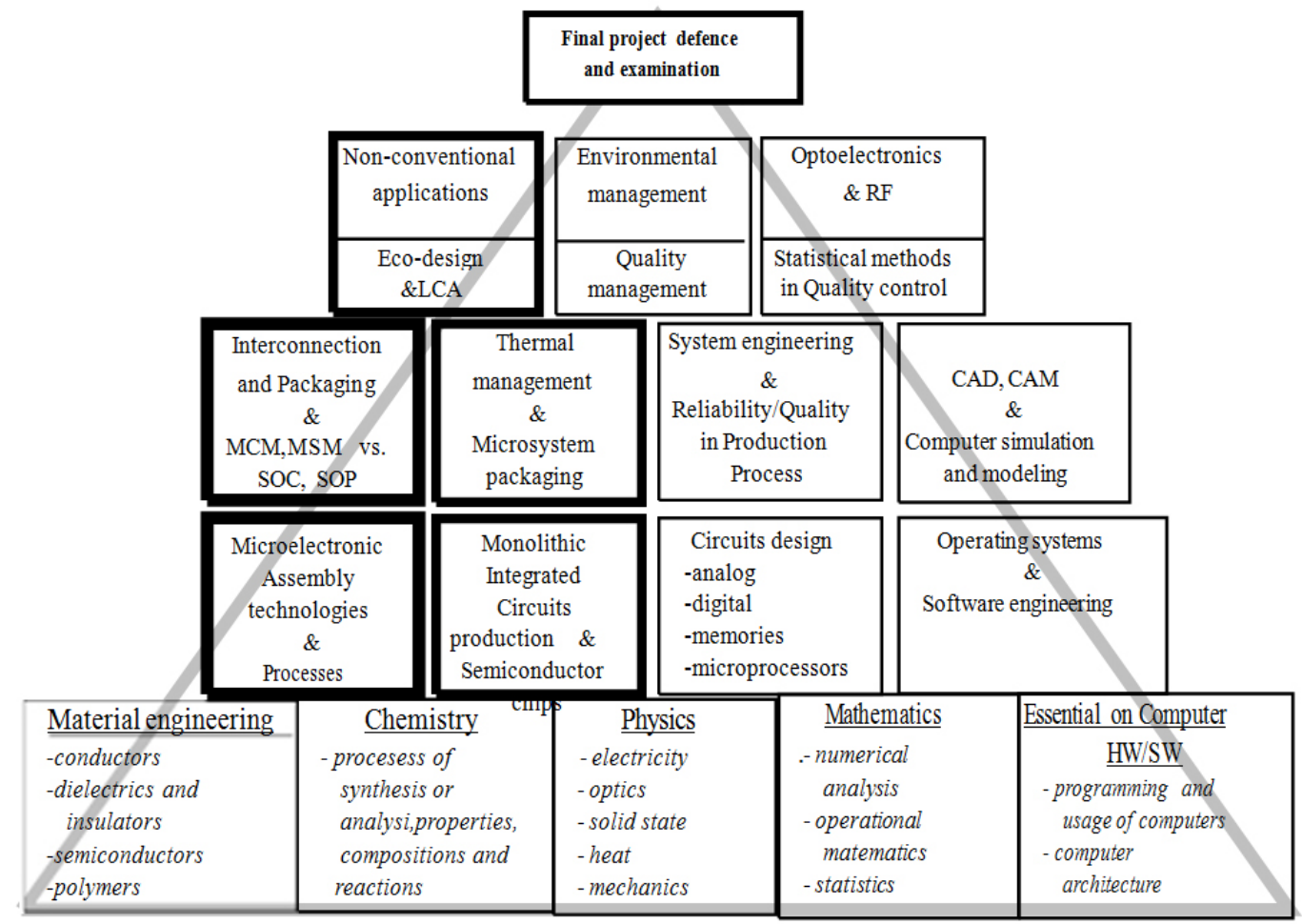

Figure 3. Basic structure of the microelectronics education system 
The base is founded by physics, chemistry and mathematics, which are the foundations of science already in secondary schools.

Core thematic topics are:

- Microelectronic Assembly Technologies and Processes

- Interconnection and Packaging including Electronics Packages

- Microsystems Packaging and Non-conventional applications

\section{Curriculum for electronics hardware education}

The main objective of this course is to teach students to orient and manage in electronics hardware. That is why the theory is strongly linked to experimental teaching. That is the only way to teach students the basic skills such as soldering, fault diagnosis and general troubleshooting. The course focuses on current and future electronics assembly and packaging technology process, which means all the sequential process steps from the semiconductor chips, through assembly processes, handling, use and repair of hardware (PC, mobile phones, displays, various control devices and measuring equipment products etc.). This knowledge of passive and active components, switches, connectors, batteries, displays, etc., can play a key role in the final decision, and thus contribute to technical and economic success. On that basis, a Curriculum for modern microelectronics technology training focused on hardware (Fig.4) has been prepared which has the following contents:

- Introduction to Electronics technology, Hardware and Substrates for Microelectronics Assembly

- Semiconductor Chips and its Performance in context of interconnection and packaging

- Passive Elements including Embedded, its Performance and Selection

- Thick \& Thin Film Technologies and Hybrid Integrated Circuits (HIC)

- Assembly Technologies for Electronic Circuits-Surface Mount Technology (SMT), Low Temperature Cofired Ceramics (LTCC)

- Electronic Packaging - Fundamentals of Electrical Design and Thermal Management

- Soldering as decisive Operation for Electronic Systems Reliability

- Quality Engineering and Total Quality Management (TQM), Statistic Process Control (SPC), Directives-WEEE, RoHS, EuP, CE

- Life Cycle Assessment, Eco-design and Electronics Impact on Environment

- Unconventional applications implemented by non-vacuum processes

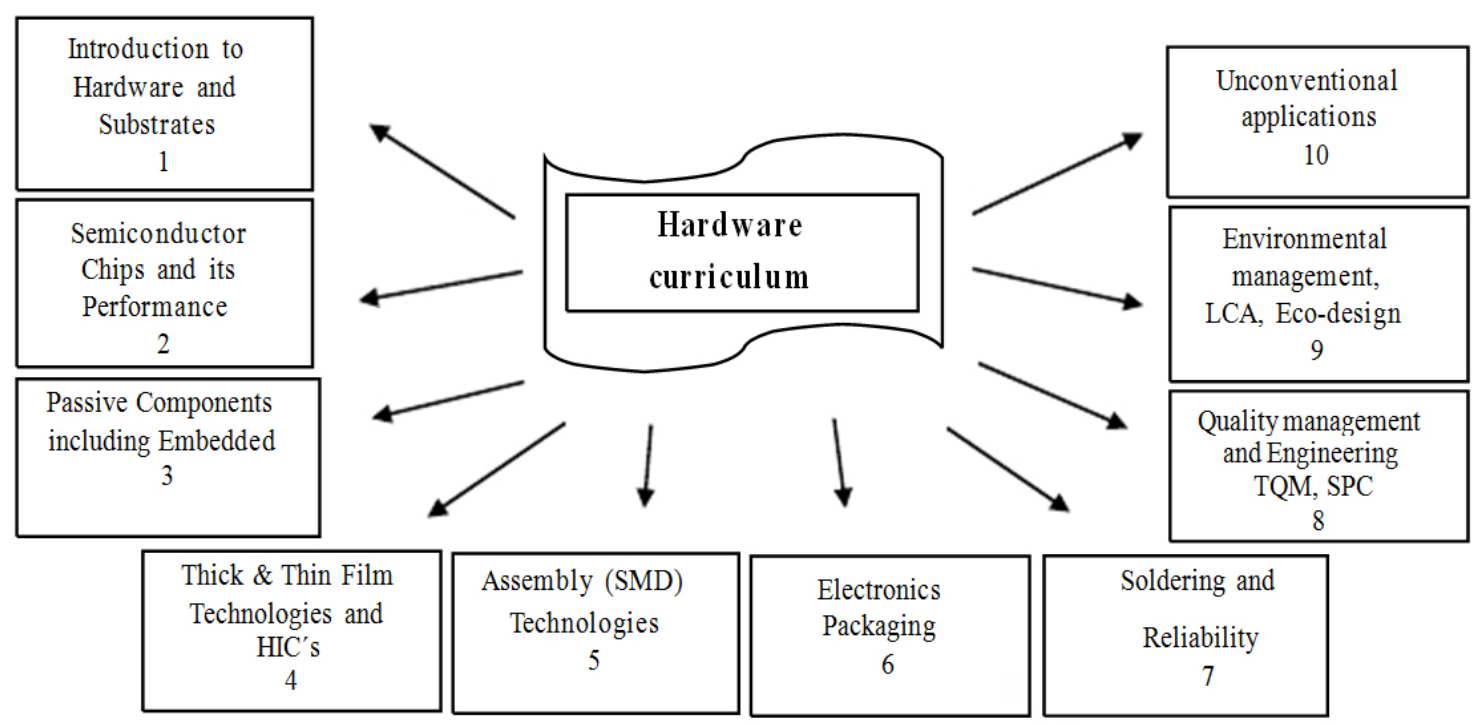

Figure 4. Syllabus of Microelectronics Technology regarding "Electronics hardware" 
This programme is arranged in two levels, for Bachelor study (Microelectronics Technology and Components), and for Master study (Modern Microelectronics Technologies). Both courses are organized in one semester period with weakly programmes consisting of one theoretical lesson (3 hours) and lab lesson every two weeks (4 hours).

The course includes twelve classroom lectures as shown in Fig. 4, where are explained the basic principles and core fundamentals to each topic. Contemporary runs laboratory exercises, which are organized in five lessons, each lasts four hours. This experimental learning is arranged in the time- and cost-constrained environment of university, where Microelectronics assembly technology lab based on non- vacuum processes is utilized. Students complete during this course in one semester the main process steps of hybrid integrated circuit realization, starting with design and finishing with packaging and testing. They learn the theoretical knowledge through own skills in practical experience. The five experimental lab exercises are following:

(1) Passive network, design of networks for main boards, screen printing, sintering, Thick Film Hybrid integrated circuits (Each student makes the design of topology of thick film hybrid integrated circuits, prepares the masks for screens and print patterns on the ceramic substrate, which are sintered in the passive network - practical skills are design, printing, sintering)

(2) Passive network trimming and evaluation, sheet resistance calculation and use of statistical tools, temperature coefficient measurement (Thick film resistors are measured and statistical evaluated, after it are trimmed in YAG laser and Temperature Coefficient of Resistivity is measured and calculated) - practical skills are touch setting resistors and their values

(3) Mounting of semiconductor chips - wire bonding, soldering and adhesive use, surface mount technology application (The main technological operation for semiconductor bare chip connection known wire bonding is demonstrated and approved, thermosonic and thermo-compression equipment is utilized, SMD are mounted by reflow soldering or by conductive adhesives) - practical skills are treatment components and their connections including soldering

(4) Thermal management analysis, ANSYS application, packaging and testing (In the first part is presented application of ANSYS software for mechanical, thermal and electrical analyzes of two basic parts of electronic components solder joints and electronics packages, in the second part is integrated circuit encapsulated by fluidization process and insulating resistivity between leads is measured) - practical skills are adoption soldering and packaging, simulation in ANSYS for microelectronics hardware with a basic knowledge of physics (Newton and Hooke's Law)

(5) Circuit testing, quality implementation (setting and measuring control parameters) in electronics production process and tools, Eco-design application in electronics and human health, legislation (This exercise is oriented to testing, statistical evaluation, Eco- design and its methods and tools (MET matrix, TPI indicator), next is demonstrated goal and application of Life Cycle Assessment including case studies, where comparison is made of the ecological impact of various products) - practical skills are measurement inside integrated circuits, understanding the importance of quality and environment management

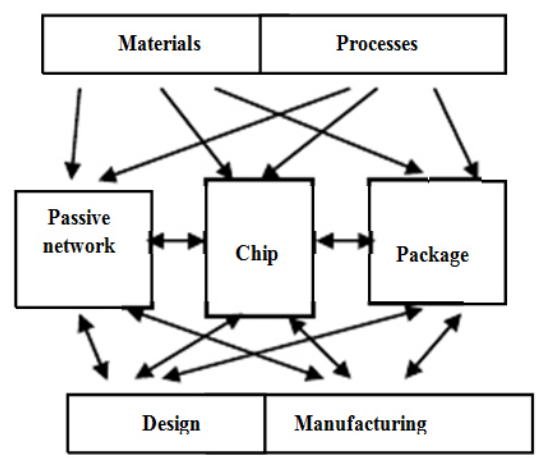

a)

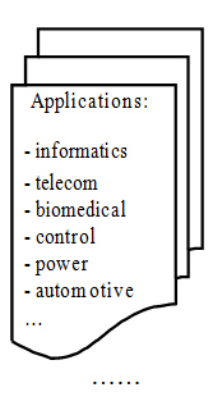

b)

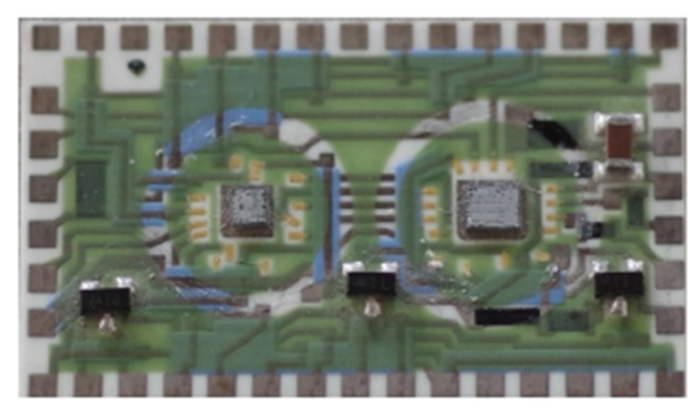

Figure 5. Schematic design of lab exercises process (a) and realized IC (b)

After the complete realization process students take in the final lab exercise one functional HIC oneself (Fig. 5). Some of experiments or demonstrations may be conducted in virtual form, which is organized on the base of e-learning [3]. Students have in experimental part good opportunity to improve their practical skills during complete process of 
integration of simple electronic circuits which makes the base of electronics system. All technological operations in the lab are based on non-vacuum processes, where the main technology is thick film realized on ceramic substrate. During experimental work students teach, probe and verify fundamentals of microelectronics and improve basic theoretical knowledge. They also learn to understand relationships and links between design and production phase, and also the main aspects that influence quality and cost. All subject matter is completed with environmental aspects.

Most of the theoretical hardware issues can only be appreciated if students have a chance to work in the laboratory. To create a demanding laboratory for just one course would be uneconomic. Fortunately the lab of Microelectronics Assembly Technology at FEEC in BUT is built systematically, and the scientific research work is utilized also for diploma theses, and is planned also for CEITEC, where strong reverse collaboration is proposed [4], [5].

\section{Conclusion}

Two main objectives are presented in this paper. The first is the presentation of a new curriculum for electronics hardware education with supporting lab exercises, which offers for students the possibility to receive practical skills in the field of modern electronics technology, including the design and building of their own hybrid integrated circuits. The basic process for experiments is thick film technology that allows realization of various electronics components including hybrid integrated circuits [6]. The lab is non-vacuum and arrangement of the basic line is not expensive - the estimated cost should be around 50 kEUR. The second scope is the necessity to collaborate with research institutions and industry. The fact that the laboratory specializes in non-vacuum packaging and interconnect technology (including mounting of bare chips of IC 's), is requested by industry, particularly from small and medium-sized enterprises. This step is supported through strong collaboration with CEITEC and IMAPS (International Microelectronics, Assembly and Packaging Society). The layout and organization of laboratory also offers some special services in this area, not only for students of all disciplines, but also for companies [7]. The lab is systematically built as multi-purpose lab where main goals to achieve are:

- educate and provide hands-on research experience for electronics engineers and managers in electrical area to take responsibility for future of microelectronics industry,

- provide quality and timely research on microelectronics topics, such as electronics assembly, interconnection and packaging,

- provide assistance to all students and the industry for their immediate needs.

The general output is to learn the ability to navigate, especially in both technical and managerial skills in electronics hardware solutions. A manager who knows how it works, what are the basic principles of electronics hardware, has knowledge about the reliability, quality and impact on the environment (eco-design) may choose a better solution than one without such understanding. A better solution will also result in a better economic outcome. This knowledge helps to manage decisions about electrical / electronic equipment throughout its life cycle, which gives positive results in reducing costs, including energy savings, reduced environmental impact, and many others.

\section{Acknowledgements}

Funding for this research was obtained through grant project BD 18709001 FEKT-S-17-3934 "Utilization of novel findings in micro and nanotechnologies for complex electronic circuits and sensor applications" supported by Ministry of Education, Sport, Youth, Czech Republic.

\section{References}

[1] International Microelectronics Assembly and Packaging Society, http://www.imaps.org, http://www.imaps.cz

[2] R. Tummala: Fundamentals of Microsystems Packaging, McGraw-Hill, New York, London, Sydney, Madrid, Lisbon, Milan, San Juan, Seoul, Mexico City, Toronto, 2001

[3] C. Vasko, I. Szendiuch, M. Novotny: Virtual Laboratory of Microelectronic Mounting and Packaging, Proc. International Conf. on Engineering Education, 3 - 7 September, 2007, Coimbra

[4] CEITEC - Central European Institute of Technology, http://www.ceitec.eu/

[5] R. Vrba: A Centre of Excellence to improve the Quality of Life and Human Health, Proceedings of 18th European 
Microelectronics and Packaging Conference, p. 10-17, 12th - 15th September, 2011, Brighton, United Kingdom

[6] Ch. A. Harper,: Electronic Packaging and Interconnection Handbook, McGraw-Hill New York, London, Sydney, Madrid, Lisbon, Milan, San Juan, Seoul, Singapore, Sydney, Toronto, 2005

[7] South Moravian Innovation Centre, http://www.risjmk.cz/userfiles/file/BRN/Szendiuch\%202012.pdf

\section{Authors}

Ivan Szendiuch holds a Master degree in Electrical Engineering from the Brno University of Technology and PhD degree the same. He joint Tesla Company 1n 1969 and in period 1992-2008 he was managing director in Vemer Ceska Group Company. In 1990 he became Assoc. Professor and in 2007 received in San Jose Fellow IMAPS, actually is president of IMAPS Czech and Slovak chapter. He is author of 5 books and many papers worldwide. At present he teaches Microelectronic Technology at the Brno University of Technology. 